\title{
DYNAMICS OF INACTIVATED SARS-COV-2 VACCINE ANTIBODY RESPONSE INSARS-COV-2-SEROPOSITIVE AUTOIMMUNE RHEUMATIC DISEASE PATIENTS
}

Nádia Emi Aikawa1 ${ }^{1, *}$, Léonard de Vinci Kanda Kupa ${ }^{1}$, Sandra Gofinet Pasoto ${ }^{1}$, Ana Cristina de Medeiros Ribeiro', Emily Figueiredo Neves Yuki ${ }^{1}$, Carla Gonçalves Schahin Saad ${ }^{1}$, Tatiana Pedrosa ${ }^{1}$, Ricardo Fuller ${ }^{1}$, Samuel Katsuyuki Shinjo ${ }^{1}$, Percival Degrava Sampaio-Barros ${ }^{1}$, Danieli Castro Oliveira de Andrade ${ }^{1}$, Rosa Maria Rodrigues Pereira ${ }^{1}$, Luciana Parente Costa Seguro ${ }^{1}$, Juliana Miranda de Lucena Valim ${ }^{1}$, Filipe Waridel ${ }^{1}$, Ana Marli Christovam Sartori ${ }^{1}$, Alberto José da Silva Duarte ${ }^{1}$, Leila Antonangelo ${ }^{1}$, Ester Cerdeira Sabino ${ }^{1}$, Paulo Rossi Menezes ${ }^{1}$, Esper Georges Kallas ${ }^{1}$, Clovis Almeida da Silva ${ }^{1}$, Eloisa Bonfa ${ }^{1}$

1.Universidade de São Paulo, São Paulo (SP), Brazil.

*Corresponding author: nadia.aikawa@gmail.com

\section{BACKGROUND}

Limited information is available on response to COVID-19 vaccines in autoimmune rheumatic disease patients (ARD) previously exposed to the SARS-CoV-2. We compared the dynamics of vaccine induced antibody production after immunization with CoronaVac in SARS-CoV-2-seropositive ARD patients (ARD+) with two age/sex balanced groups: SARS-CoV-2-naïve ARD patients (NAÏVE-ARD) and SARS-CoV-2-seropositive control group (CTRL+).

\section{METHODS}

Participants of this phase 4 prospective controlled study (clinicaltrials.gov \#NCT04754698) were vaccinated with two doses of CoronaVac (28-days interval). Primary objective was immunogenicity dynamics evaluated by median neutralizing activity (NAb-activity)/anti-SARS-Cov-2 $\ln (\lg G)$ titers $[\ln (\lg G)]$ from D0-D28 and from D28-D69. Secondary objectives included safety and other immunogenicity parameters.

\section{RESULTS}

Diseases and therapy were similar in ARD+ and NAÏVE-ARD groups ( $p>0.05$ ). Comparable dynamics were observed for $A R D+$ and CTRL+ with a plateau increase occurring from D0-D28 (ARD+, NAb-activity: 59.1 to $81.8 \%$, mean difference $-12.1 \%$, $\mathrm{p}=0.002$ and anti-S1/S2-GMT: 52.3 to 128.9, In (IgG) mean difference -0.9, $p<0.001$ ) and (CTRL+, NAb-activity: 57.5 to $91.9 \%$, mean difference $-25.2 \%, p<0.001$ and anti-S1/S2-GMT: 53.3 to 202.0, $\ln (\lg G)$ mean difference $-1.33, p<0.001)$. Insignificant increments occurred from D28-D69 for ARD+ and CTRL+ regarding NAb-activity $(p>0.999)$ and anti-S1/S2-GMT $(p<0.999)$. In contrast, a distinct pattern was observed for NAÏVE-ARD with negligible increase from D0-D28 (NAIIVE-ARD, NAb-activity: 15 vs. $15 \%$, mean difference $-8.3 \%, p<0.001$ and anti-S1/S2-GMT:2.3 vs. 5.7, $\ln (\mathrm{IgG})$ mean difference $-0.93, p<0.001)$ and a moderate increase from D28-D69 (NAÏVE-ARD, NAb-activity: 15.0 vs. 39.4\%, mean difference -19.2\%, p < 0.001 and anti-S1/S2 - GMT: 5.7 vs. 29.6, $\ln (\lg G)$ mean difference $-1.65, p<0.001$ ) (Table 1, Figure 1). Supporting these findings, significant differences in NAb activity/In(IgG) anti-S1/S2-GMT were observed between ARD+ vs. NAIIVE-ARD at D0:43.8\%/3.14, p<0.001, D28: 47.5\%/3.12, $p<0.001$ and D69: 29\%/1.53, $p<0.001$, whereas no difference occurred between ARD+ vs. CTRL+ at D0: $-0.5 \% /-0.02$, $p>0.999$ and $D 69:-12.3 \%, p=0.167 / 0.32 \%, p=0.258$ with minor difference at D28: $-13.6 \%, p=0.067 /-0.45, p=0.006$. 
Table 1. Median percentage of neutralizing activity and anti-SARS-CoV-2 S1/S2 IgG titers before(D0) and after the first(D28) and second(D69) doses of Sinovac-CoronaVac vaccination in ARD+, NAÏVE-ARD and CTRL+.

\begin{tabular}{|c|c|c|c|c|c|c|}
\hline & ARD+ & CTRL+ & NAÏVE -ARD & ARD+ & CTRL+ & NAÏVE -ARD \\
\hline & $n=157$ & $\mathrm{n}=157$ & $n=471$ & $\mathrm{n}=157$ & $\mathrm{n}=157$ & $n=471$ \\
\hline \multirow[t]{2}{*}{ Do } & 59.1 & 57.5 & 15.0 & 52.3 & 53.3 & 2.3 \\
\hline & $(38.8-82.5)$ & $(41.2-79.1)$ & $(15.0-15.0)$ & $(42.9-63.9)$ & $(45.4-62.5)$ & $(2.2-2.3)$ \\
\hline \multirow[t]{2}{*}{ D28 } & 81.8 & 91.9 & 15.0 & 128.9 & 202.0 & 5.7 \\
\hline & (54.3-95.8) & $(78.6-96.4)$ & $(15.0-15.0)$ & $(105.6-157.4)$ & $(174.8-233.4)$ & $(5.1-6.4)$ \\
\hline \multirow[t]{2}{*}{ D69 } & 78.7 & 92.1 & 39.4 & 137.1 & 188.6 & 29.6 \\
\hline & (57.4-94.1) & $(74.6-96.3)$ & $(15-64.8)$ & (116.2-161.9) & $(167.4-212.6)$ & $(26.4-33.3)$ \\
\hline $\mathrm{p}$ (Groups) & & $<0.001$ & & & $<0.001$ & \\
\hline $\mathrm{p}$ (Timepoints) & $<0.001$ & $<0.001$ & $<0.001$ & $<0.001$ & $<0.001$ & $<0.001$ \\
\hline $\mathrm{p}$ (Interaction) & & $<0.001$ & & & $<0.001$ & \\
\hline
\end{tabular}
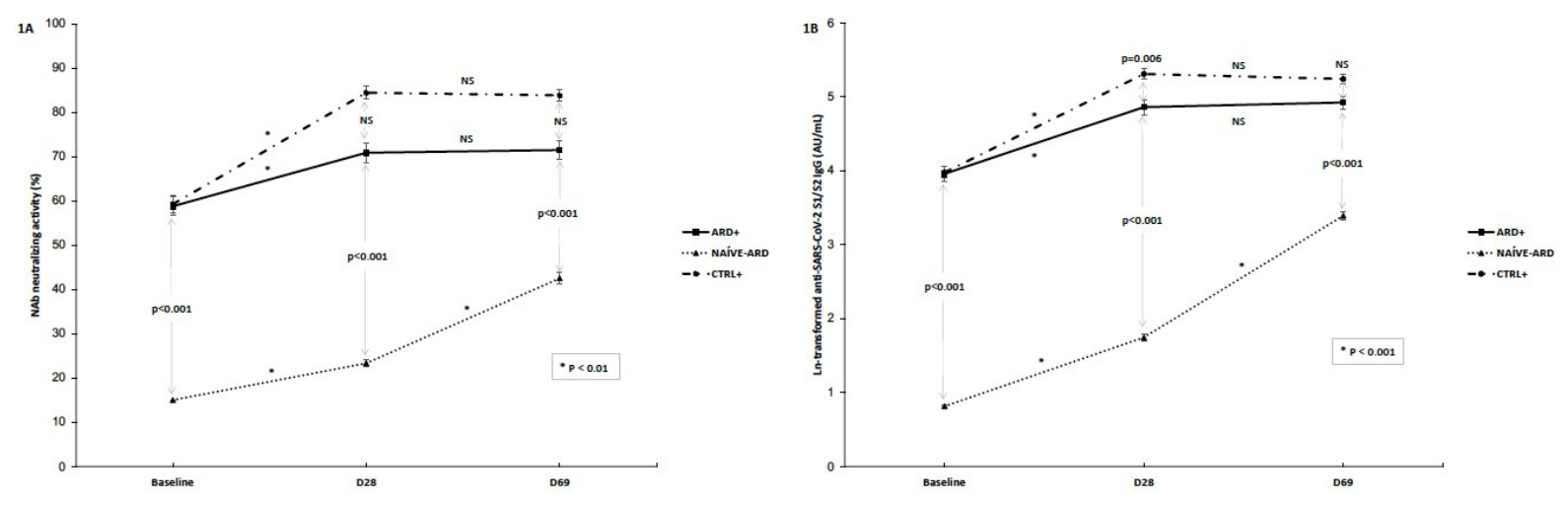

Figure 1.

\section{CONCLUSION}

ARD+ patients mount a robust plateau response after a single dose of inactivated SARS-CoV-2 vaccine, independent of preexisting ARD/therapy, whereas NAIIVE-ARD patients require the second dose to ensure a moderate antibody production. Our findings raise the possibility of a single dose regimen in ARD patients previously exposed to SARS-CoV-2.

\section{KEYWORDS}

Autoimmune rheumatic diseases, COVID-19, Vaccine, Seropositive, Immunogenicity. 